\title{
Yerba mate improves cardiovascular health in normocholesterolemic and hypercholesterolemic subjects
}

\section{Abstract}

Yerba mate is highly consumed in South America and has become a popular beverage worldwide because of its healthy properties: antioxidant, vaso-dilating, lipid-reducing, anti-glycation, anti-inflammatory and weight-reducing effects, among others. These effects have been attributed to its high content in polyphenols, mainly chlorogenic acidS, but also quercetin, kaempferol and rutin. Other components in mate are methylxhantines, saponins, vitamins, minerals and alkaloids.

The objective of this work was to evaluate the effects of regularly consuming a moderate, realistic amount of yerba mate ( 3 beverages/day) on cardiovascular health and anthropometric parameters, in healthy and cardiovascular risk subjects.

A randomized, crossover, controlled study was performed in normocholesterolemic $(\mathrm{NC}, \mathrm{n}=25)$ and hypercholesterolemic $(\mathrm{HC}, \mathrm{n}$ =27), non-smoker, non-vegetarian, men and women, with BMI $18-25 \mathrm{~kg} / \mathrm{m}^{2}$. After a run-in stage, the effects of consuming three servings/day of mate during 8 weeks (mate stage) were compared with an isotonic drink during the same time (control stage). Along the study, polyphenol rich foods were restricted. At the beginning and the end of each intervention, blood pressure was measured, urine and blood samples were collected. Triglycerides (TG), total cholesterol (TC), high-density lipoprotein (HDL), low-density lipoprotein (LDL) and very-low-density lipoprotein (VLDL) cholesterol concentrations were measured. Dietary records and physical activity questionnaires were completed in each stage. Results were statistically studied using a mix model with repeated measures and Bonferroni test (SPSS 23.0).

The regular consumption of yerba mate decreased blood lipid concentrations [TC $(p<0.001)$, LDL $(p=0.001)$, TG $(p=0.027)]$ and blood pressure [systolic $(\mathrm{p}<0.001)$, diastolic $(<0.001)$ ] in both groups, with greater effects in HC than NC. Thus, it may be concluded that moderate consumption of yerba mate reduces cardiovascular risk, particularly in hypercholesterolemic subjects.

Funded by the Spanish State Research Agency, projects AGL2010-18269 and AGL2015-69986-R

\section{Conflict of Interest}

There is no conflict of interest 